\section{Andrea Diehl}

\section{Das Levico-Wasser: Quelle des Wohlbefindens}

Erstmals wurde das wirksame Heilwasser der Levico-Quelle in Vetriolo, Trentino/Italien, im Jahr 1856 von dem Bergbauern Domenico Zen entdeckt. Danach wurde bereits sehr rasch die hohe Wirksamkeit des Heilwassers an der heimischen Bevölkerung beobachtet, und die ersten chemischen Analysen wurden 1858 durch den italienischen Arzt Professor Luigi Manetti durchgeführt. Bereits hier zeigte sich die wertvolle und einzigartige Zusammensetzung des «starken Wassers».

Neben dem in kolloidaler Lösung befindlichen Eisen und Kupfer konnten viele weitere Substanzen nachgewiesen werden. Levico-Wasser enthält zudem Zink, Kalzium, Magnesium und Arsen, das hier - an proteinhaltige Stoffe in kolloidaler Form gebunden - nicht giftig ist.

Innerhalb weniger Jahre entwickelte sich eine Anwendung als Trink- und Badekur, sodass bereits 1863 mit ca. 10000 Bädern und 700 Gästen pro Saison aufgewartet werden konnte.

Als Heilmittel zur pharmazeutischen Anwendung konnten die anthroposophischen Heilmittel-Hersteller Weleda und Wala Levico-Wasser als potenziertes Mittel in Tropfen-, Globuli- und Ampullenform konzipieren.

Das Herzstück einer Bäderkur beruht auf den täglichen Levico-Bädern in unterschiedlicher, individueller Dosierung nach ärztlicher Anleitung. Über die transdermale Resorption können die Mineralstoffe aufgenommen werden.

Nach Abschluss der Thermalanwendung können die Kuren durch orale

\title{
Levico-Heilwasser bei Erschöpfung, Rekonvaleszenz und Wechseljahresbeschwerden
}

oder inhalative Aufnahme sowie durch Injektionen fortgeführt und prolongiert werden.

Zum Einsatz kommt Levico-Wasser bei allen Formen der Erschöpfung und Rekonvaleszenz. Hierzu zählen berufliche, soziale, seelische und körperliche Erschöpfungen unterschiedlichster Graduierungen - beispielsweise nach rezidivierenden Infekten, bei allen Formen von Wechseljahresbeschwerden, bei hormonellen Dysregulationen, bei Eisenmangelanämie, als Tumorbegleittherapie usw.

Durch die langjährige Erfahrung konnte das Ärzteteam des Wellness-Hotels Casa di Salute in Roncegno feststellen, dass bei den Patienten nach einer zwei- bis dreiwöchigen Initialkur durch regelmässige Levico-Intervallanwendungen mit Kurzkuren in den Folgejahren eine Gesundheitsstabilisierung erzielt werden kann.

\section{Fallbeispiel}

- 64-jährige Patientin, selbstständig als Heileurythmistin tätig, geschieden, ein Sohn (26 Jahre).

- Im Jahr 2012 erstmals ambulanter Kontakt. Nach enorm hoher familiärer Belastung mit Trennung und Umzug schilderte sie folgende Symptome: Gewichtsabnahme, Reizdarm mit Durchfällen und Meteorismus, nächtliches Erwachen, häufiges Schwitzen, extreme Erschöpfung mit hoher Pausenbedürftigkeit, leichte depressive Verstimmung.

- 2012 zeigte sich in der Labor-, Ultraschall- und Endoskopieuntersuchung kein Anhalt für eine Tumorerkrankung. Im Labor zeigte sich ein deutlicher Eisen-, Vitamin-D-, Zink- und Selenmangel.

- 2013 erfolgte eine dreiwöchige LevicoBäderkur. Danach war eine deutliche Stabilisierung mit Ausgleich aller Laborwerte, Gewichtszunahme und seelischer Harmonisierung feststellbar. Die Patientin fühlte sich vollends umhüllt durch die Levico-Bäder. Nach anfänglicher Ermüdung durch die Bäder, was durchaus einen typischen Verlauf darstellt, zeigte sich zunehmend ein positiver Verlauf mit deutlicher Verbesserung der Vitalität, nächtlichem Durchschlafen ohne Schwitzattacken und Regulierung der Darmtätigkeit. Die Kur wurde zuhause durch Levico-compGlobuli (Wala) verlängert. Auch die Laborkontrollen zeigten nach 6 Wochen eine völlige Normalisierung.

- In den Jahren 2014 und 2015 kam die Patienten zu weiteren Kurzkuren. Sie bestätigt die anhaltenden Erfolge bis zum heutigen Tag.

\section{Fazit}

Der im Fallbericht geschilderte Verlauf ist typisch für eine Vielzahl von Fällen. In unserer heutigen Zeit stellt das Levico-Wasser eine hervorragende Möglichkeit dar, regulierend auf den Gesamtorganismus einzuwirken, und hat ein breites Therapiespektrum. Die Patienten selbst sind oft überrascht über die tiefgreifende Wirkung und schätzen die Anwendung über viele Jahre hinweg.

\section{KARGER}

(c) 2017 S. Karger GmbH, Freiburg

Fax +497614520714
Andrea Diehl

Fachärztin für Allgemeinmedizin und anthroposophische Medizin

Försterstrasse 26, 66111 Saarbrücken, Deutschland

info@ arztpraxis-andreadiehl.de 